\title{
Effect of Atmosphere on Collinear Double-Pulse Laser-Induced Breakdown Spectroscopy
}

\section{6th International Conference on Laser- Induced Breakdown Spectroscopy}

\author{
Andrew J. Effenberger, Jr. \\ Jill R. Scott
}

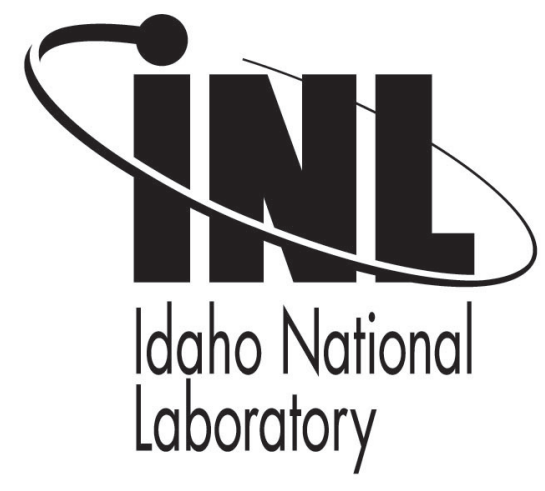

This is a preprint of a paper intended for publication in a journal or proceedings. Since changes may be made before publication, this preprint should not be cited or reproduced without permission of the author. This document was prepared as an account of work sponsored by an agency of the United States Government. Neither the United States Government nor any agency thereof, or any of their employees, makes any warranty, expressed or implied, or assumes any legal liability or responsibility for any third party's use, or the results of such use, of any information, apparatus, product or process disclosed in this report, or represents that its use by such third party would not infringe privately owned rights. The views expressed in this paper are not necessarily those of the United States Government or the sponsoring agency. 


\title{
Effect of Atmosphere on Collinear Double-Pulse
}

\section{Laser-Induced Breakdown Spectroscopy}

\author{
Andrew J. Effenberger Jr. and Jill R. Scott* \\ Idaho National Laboratory (INL), Idaho Falls, ID 83415
}

Keywords: DP-LIBS, Helium, Argon, Air, Reduced Pressure

Short Running Title: Effect of Atmosphere on Collinear DP-LIBS

*Address correspondence to:

Dr. Jill R. Scott

Idaho National Laboratory

MS 2208

1725 N. Yellowstone HWY

Idaho Falls, ID 83415

Tel.: 208-526-0429

Fax: 208-526-8541

Email: jill.scott@inl.gov 


\section{Abstract}

Double-pulse laser-induced breakdown spectroscopy (DP-LIBS) has been shown to enhance LIBS spectra. Several researchers have reported significant increases in signal-to-noise (S/N) and/or spectral intensity compared to single-pulse (SP) LIBS. In addition to DP-LIBS, atmospheric conditions can also increase sensitivity. Thus, in this study, a collinear DP-LIBS scheme was used along with manipulation of the atmospheric conditions. The DP-LIBS scheme consisted of an initial $45 \mathrm{~mJ}$ pulse at $1064 \mathrm{~nm}$ fired into a sample contained in a controlled atmospheric/vacuum chamber. A second analytical $45 \mathrm{~mJ}$ pulse at $1064 \mathrm{~nm}$ was then fired 0 to $200 \mu$ s after and along the same path of the first pulse. Ar, He, and air at pressures ranging from atmospheric pressure to 1 Torr are introduced during DP-LIBS and SP-LIBS experiments. For a brass sample, significant increases in the spectral intensities of $\mathrm{Cu}$ and $\mathrm{Zn}$ lines were observed in DPLIBS under Ar compared to DP-LIBS in air. It was also found that $\mathrm{Cu}$ and $\mathrm{Zn}$ lines acquired with SP-LIBS in Ar are nearly as intense as DP-LIBS in air. While collinear DP-LIBS is effective for increasing the sensitivity for some reduced atmospheres (i.e., Ar and air at 630 to 100 Torr and He at 300 Torr), the enhanced spectral intensity ultimately dropped off as the pressure was reduced below 10 Torr for all atmospheric compositions in the experimental arrangement used in this study. At all pressures of air and Ar, the plasma temperature remained rather constant with increased inter-pulse delays; however, the plasma temperature was more variable for different He gas pressures and inter-pulse delays. 


\section{Introduction}

Laser-induced breakdown spectroscopy (LIBS) is a spectroscopic technique that uses a focused, pulsed laser to form a plasma in air as well as in or on liquid and solid surfaces [1-9]. By analyzing the emission spectra from the LIBS plasma, the atomic composition of an analyte can be determined. No sample preparation is necessary for LIBS, making it a very popular technique in many applications that include environmental monitoring [10-12], material analysis [13], forensics [14], characterization of fossils [15], biological identification [16,12], analysis of works of art [17], and space exploration $[18,19]$. Though the applications of LIBS are vast, LIBS suffers a disadvantage in sensitivity when compared to other spectroscopy techniques, such as inductively coupled plasma atomic emission spectroscopy (ICP-AES) [20-24].

Double-pulse laser-induced breakdown spectroscopy (DP-LIBS) is typically used to improve sensitivity compared to single-pulse LIBS (SP-LIBS) [25]. DP-LIBS involves the use of two laser pulses, either collinear or orthogonal, that are separated temporally on the order of nano- or microseconds. An orthogonal configuration uses a non-ablative pulse parallel to the surface that is fired before [26-30,1,31-36]. Using an orthogonal DPLIBS configuration with a non-ablative pulse fired above the surface followed by an analytic/ablation pulse, Stratis et al. found a 20 -fold improvement in LIBS spectral intensity compared to SP-LIBS on glass [34]. Scaffidi et al. used an orthogonal DP-LIBS method with a pre-pulse fired into the sample first [33]. The first laser pulse was generated from a femtosecond pulse-width laser and was followed by a nanosecond pulse-width laser (the analytical pulse) fired above the surface through the ablation plume created from the first pulse. This femtosecond-nanosecond method resulted in significant 
30-fold increase in spectral intensity. In a collinear configuration, both laser pulses are fired along the same path and the LIBS measurement is made from the plasma generated by the second laser pulse. Sattmann et al. used this method, which resulted in 2-fold increase in spectral intensity and a 7-fold increase in ablation [37]. Both orthogonal and collinear DP-LIBS can improve spectral intensity and signal-to-noise; however, depending on the available equipment and application, one or the other maybe preferred.

It has been noted in the literature that the mechanisms that lead to signal enhancement in DP-LIBS are not clear [38,39] and some proposed mechanisms are often contradictory [40]. While the DP-LIBS process is highly complex, there are three factors that appear to be of primary importance in producing signal enhancements: 1) pulseplasma coupling energetics between first LIP and the second laser pulse and LIP; 2) sample heating in the context of the plasma produced by the first laser pulse; and 3) local atmospheric pressure and number density effects [40,39]. A simplified description of the collinear DP-LIBS process starts when the first laser pulse interacts with the sample matrix beginning the sample heating and ablation process. The first LIP then freely expands into the cold environment of the buffer gas [40]. This expansion is similar to that postulated for SP-LIBS that would involve a low pressure region with forward particle flow, in the direction of the ablating laser pulse that is usually normal to the sample surface [41]. The first stage is followed by a transition regime with momentum transferred to the buffer gas forming a shock wave that would be followed by a diffusion stage at higher gas pressure. The result would be a confined, sphere-like structure for the first LIP or SP-LIP [42]. During the expansion, the first LIP loses some energy in the process of ionizing the buffer gas. The second laser pulse in DP-LIBS interacts with the 
heated environment of the first LIP and sample created by the first laser pulse. The preheated target surface produced by the first laser pulse and LIP reduce the breakdown threshold as well as to reduce the background gas density behind the shock wave of first LIP [43], which reduces the laser shielding for the second laser pulse [26]. The rarefied gas and higher temperature produced by the first LIP creates an environment that leads to a faster and greater expansion of the plume for DP-LIBS compared to SP-LIBS $[40,36]$. Expansion of the second LIP in the rarefied gas created by the first LIP saves some of its energy, leading to an increase of the emission signal. DP-LIBS signal enhancements include increased detection time and emission volume that result from a combination of effects $[39,25]$.

There also appears to be agreement that the DP-LIBS mechanisms are dependent on the inter-pulse delay. For example, more efficient ablation is observed for inter-pulse delays less than a few hundred nanoseconds. This is rationalized because of the preheated sample and rarefied atmosphere in front of the target created by the first laser mentioned above that reduce the breakdown threshold $[38,40]$. However, with longer delays, the sample has time to equilibrate with the environment produced by the first laser pulse and there is less increase in mass ablation. Galbacs et al. [43] have suggested that this mechanism may last through inter-pulse delays as long as $10 \mu \mathrm{s}$. Another plasma condition that varies with inter-pulse delay is the plasma temperature, which is higher with DP-LIBS and leads to longer lifetimes; however, the effect gets averaged out with integration over the whole plasma volume [40] and with longer inter-pulse delays [38]. Thus, longer inter-pulse delay times between development of the first LIP and the second laser pulse tend to negate effects observed with short, nanosecond inter-pulse delays. 
In addition to DP-LIBS, atmospheric conditions (i.e., pressure and gas composition) also impact the observed spectral intensity $[44,45]$. Gas composition also influences LIBS spectra and can be used to increase signal intensity. Results reported by Iida [45] revealed that that He and Ar atmospheres were both effective for increasing LIBS spectral intensity compared to air at one atmosphere ( $\sim 60$ Torr). At higher pressures, there are more collisions with the buffer gas, which leads to decreased plasma lifetime, but an enhancement of energy transfer from buffer gas to analyte [46]. Heavier buffer gases due slow plume expansion resulting in shorter plasma length and better plasma confinement $[47,48]$. The mass ablation can also be affected by the gas composition. While the ablation rate dropped in the order $\mathrm{He}>\mathrm{Ne}>$ air $>\operatorname{Ar}[49]$, reported the signal intensities were found to decrease in the order of $\mathrm{Ar}>\mathrm{Ne}>\mathrm{He}$. These observations were attributed to stronger plasma excitation in argon. Similar to DP-LIBS, expansion of a laser produced plume in a background or buffer gas is also complicated and difficult to model thoroughly $[41,50]$. This can be further complicated when changes in pressure are included [46]. Reduced pressures have been used to increase spectral resolution and signal-to-noise $(\mathrm{S} / \mathrm{N})$, but often at the expense of signal intensity $[51,44]$. However, Iida [45]found that as pressure was decreased to 100 Torr, LIBS spectral intensity increased for both Ar and air buffer gases. If pressure is decreased too far, then the LIBS intensity tends to erode because of the lack of plasma confinement. At reduced pressures, the plasma expansion is more elongated [52]. The spectral intensity increase at low pressures is likely due to reduced plasma shielding, resulting in more ablation as well as reduced Stark broadening [44]. Similar to DP-LIBS, the mechanisms responsible for 
the observations related to gas composition and pressure are not fully understood, partly because of the variations in experimental conditions [44].

In this study, a collinear DP-LIBS scheme was used along with manipulation of the atmospheric conditions to investigate the potential for further improvements in sensitivity. Since the second laser pulse in the orthogonal DP-LIBS method is parallel to the surface, it would become progressively more difficult for the second laser pulse to intersect the laser-induce plasma (LIP) created by the first laser pulse because as the atmospheric pressure is reduced the plume elongates along the path of the first ablative laser pulse [52]; thus, a collinear arrangement was employed in this study. The effect of DP-LIBS was evaluated in Ar, He, and air at pressures ranging from atmospheric pressure to 1 Torr. For evaluation purposes, the DP-LIBS results were compared with those for SP-LIBS experiments under the same atmospheric conditions.

\section{Experimental}

The collinear DP-LIBS experimental set-up with atmospheric chamber is illustrated in Fig. 1. Two laser pulses, both at $1064 \mathrm{~nm}$, were combined into the same beam path with a beam splitter. A Continuum Powerlite II Nd:YAG (Santa Clara, CA) supplied the first pulse (45 mJ) and a Continuum Surelite I Nd:YAG (Santa Clara, CA) supplied the second pulse $(45 \mathrm{~mJ})$. The timing between the two pulses was controlled with a delay generator and monitored with an oscilloscope from the laser Q-switch. A single-pulse (SP) is assumed when the inter-pulse delay is $0 \mathrm{~s}$, which would provide the equivalent total laser energy produced by DP-LIBS $(90 \mathrm{~mJ})$. The laser pulses were focused onto a brass sample housed in a controlled atmospheric/vacuum chamber, which 
was purged with air, Ar, and He. The pressure of the bath gases was varied from atmospheric (630 Torr at an elevation of $1430 \mathrm{~m}$ ) to 1 Torr, which was measured using a pressure gauge. To minimize changes related to laser ablation depth, the sample was moved between experiments using a x,y-translation stage.

LIBS measurements were taken perpendicularly to the plasma expansion along the in the direction of the incident laser beam (i.e., perpendicular to the sample surface). The optical collection geometry was chosen for efficient plume coverage with a field of view (FOV) of up to 1-in ( $\sim 25 \mathrm{~mm})$ from the target surface. To obtain the most representative light collection as possible, the sample desorption location was set at the focal length of the lens to minimize divergence. The collection angle of the fiber optic bundle with a numerical aperture (NA) of 0.22 is $25.4^{\circ}$. A fused silica lens with 2-in diam. with an $85 \mathrm{~mm}$ focal length and NA of 0.299 was used that translates into a total collection angle of $34.8^{\circ}$, which is greater than the acceptance angle of the fiber optic. Therefore, the sample location was set at approximately mid-point of the lens. This geometry was used to ensure that light from the sample was within the acceptance angle of the fiber optic bundle (lens half-angle $17.4^{\circ}$ ). Fiber optic was located at approximately the focal length of lens $(85 \mathrm{~mm}$ ). Because the quartz vacuum window (thickness of $0.105 \mathrm{~mm}$, refractive index 1.4585) bends the light in slightly, the fiber optic location was adjusted to optimize signal.

The fiber optic was coupled to an Acton SpectraPro ${ }^{\circledR}$ 500i Czerny-Turner spectrometer (Acton, MA) equipped with a Princeton Instruments PI-max 512 x 512 pixel ICCD camera (Trenton, NJ). The spectrograph used an $1800 \mathrm{~g} / \mathrm{mm}$ grating for measurements of the $481 \mathrm{~nm}$ zinc line and a $150 \mathrm{~g} / \mathrm{mm}$ grating for broadband 
measurements. The inter-pulse delay of the DP-LIBS experiment was varied from $0 \mu$ s to $120 \mu \mathrm{s}$. The ICCD was operated with a $20 \mu$ s gate width and a delay of $1 \mu$ s after the firing of the second laser pulse. SP-LIBS experiments were conducted by setting the inter-pulse delay to $0 \mathrm{~s}$ so that the energy equivalent to the two laser pulses was preserved [26]. Winspec $/ 32^{\circledR}$, provided by Princeton Instruments, was used to control the spectrograph and camera to collect spectra. Unless specified otherwise, reported spectra are accumulations of 8 shots. Spectra were exported in ASCII and plotted in MatLab ${ }^{\circledR}$ from MathWorks ${ }^{\circledR}$ (Natick, MA). The peak area was calculated and a baseline was subtracted in MatLab ${ }^{\circledR}$.

For excitation temperature measurements the $150 \mathrm{~g} / \mathrm{mm}$ grating was used to measure the lines $510.6 \mathrm{~nm}, 515.3 \mathrm{~nm}$, and $521.8 \mathrm{~nm}$ from $\mathrm{Cu}$ emission. The peak areas from these lines were used to create a Boltzmann plot for each condition using the equation $I \mathrm{i}=g \mathrm{i} A \mathrm{i} / \lambda \exp [-E \mathrm{i} /(k T \mathrm{e})]$. In the Boltzmann equation, $I \mathrm{i}$ is the line intensity, $k$ is the Boltzmann constant, and $g \mathrm{i}, A \mathrm{i}, E \mathrm{i}$, and $\lambda$, are spectroscopic constants corresponding to the statistical weight, transitions probability, energy of the excited state and wavelength, respectively. Spectral constants found in Hafez et al. [46] were used. After rearranging and taking the natural log of the Boltzmann equation, the line intensity and constants were used to create an x,y-plot, from which the excitation temperature, Te, was determined from the slope.

\section{Results and Discussion}

The complexity of DP-LIBS and variations of LIBS results with gas composition and pressure may partly due to the experimental configurations and parameters $[44,43]$. 
Bogaerts et al. [38] noted that the trend in plasma temperature for DP-LIBS is generally reported to increase, although some reports show negligible change or even lower temps. These types of variation may depend on the experimental configuration as well as the observation times. Because of these complications, this study was conducted to survey the effects that combing DP-LIBS with various gas compositions and pressures would have on signal intensity with the current experimental setup.

A collinear DP-LIBS configuration was used in this experiment due to the simplicity of the experiment and its compatibility with LIBS plume geometry at reduced pressures. As the first laser plasma expands toward the laser source, the second laser pulse will intersect a larger portion of the first laser plasma than if an orthogonal arrangement were used. If the ablative pulse is fired first and the analytic pulse is fired second in an orthogonal configuration, the ablative plume will vary wildly in size and density similar to SP-LIBS work reported by Yalcin et al. [52]. Thus, firing a pre-ablative pulse in an orthogonal DP-LIBS configuration above a surface at reduced pressures may result in no plasma formation because plasma intensity of gases decrease with decreasing pressure in low pressure regimes (less than 400 Torr) [53]. In orthogonal DP-LIBS, the location at which the analytic pulse is fired in the plume would be another variable, making the study difficult to extract meaningful information. Therefore, a collinear arrangement was selected for this study.

SP-LIBS serves as the benchmark against which DP-LIBS results are compared. Not all DP-LIBS compare SP to DP in the same manner [40]. For an appropriate comparison of collinear DP-LIBS with SP-LIBS, it is important that the laser energy deposition for the two experiments be a similar as possible. When a single laser is used 
that can provide two pulses separated by a variable delay, the SP-LIBS has been performed either with a single laser pulse equivalent in energy to the two laser pulses [37] or with an inter-pulse delay of $0 \mathrm{~s}$ between the pulses [26]. In the present case, two different lasers are used to provide the two pulses for DP-LIBS; therefore, SP-LIBS experiments were performed with an inter-pulse delay of $0 \mathrm{~s}$ so that any variation in laser beam quality between the two lasers would be preserved and replicated for SP-LIBS. However, it should be noted that SP-LIBS is not always defined in the same manner in the literature. Sometimes it is stated that the first laser pulse is equivalent to SP-LIBS or that energy of the DP-LIBS is twice that SP-LIBS $[38,40]$. Therefore, care must be taken to clarify or know what comparison is being made when interpreting DP-LIBS versus SPLIBS results.

An atmosphere of Ar seems to result in the most intense spectra as seen in Fig. 2 that compares a DP-LIBS spectrum with an $80 \mu$ s inter-pulse delay in 630 Ar with a SPLIBS spectrum in 630 Torr air for a brass sample. The increase in intensity is related to an increase in sensitivity. For example, the ratio between the signal-to-noise $(\mathrm{S} / \mathrm{N})$ for the spectrum acquired in 630 Torr Ar with a $80 \mu$ s inter-pulse delay and the $\mathrm{S} / \mathrm{N}$ for 630 Torr air with 0 us inter-pulse delay is about $\sim 70$. In addition to an increase in intensity of the $\mathrm{Cu}$ lines at $510 \mathrm{~nm}, 515 \mathrm{~nm}$, and $521 \mathrm{~nm}$, the $\mathrm{Zn}$ lines also increased. Because $\mathrm{Zn}$ is the minor component of the brass sample $(\sim 5 \%)$, the changes in the $\mathrm{Zn}$ line at $481 \mathrm{~nm}$ were used to monitor effects of atmospheric changes and the experiments where repeated to observe changes in the $\mathrm{Cu}$ lines for temperature measurements.

In Fig. 3a, DP-LIBS in 300 Torr air with a $30 \mu$ s interpulse delay has approximately a 5-fold increase in intensity when compared to SP-LIBS in air for the 
$481 \mathrm{~nm} \mathrm{Zn} \mathrm{line.} \mathrm{Fig.} \mathrm{3b} \mathrm{plots} \mathrm{the} \mathrm{peak} \mathrm{area} \mathrm{of} \mathrm{the} 481 \mathrm{~nm} \mathrm{Zn} \mathrm{line} \mathrm{versus} \mathrm{inter-pulse} \mathrm{delay}$ at different pressures of air. It can be seen in Fig. $3 b$ that the maximum intensity is seen in 300 Torr with an inter-pulse delay of $30 \mu$ s. In general, 100 Torr, 300 Torr, and 630 Torr DP-LIBS is rather effective at increasing the spectral intensity of Zn $481 \mathrm{~nm}$ compared to SP-LIBS at these pressures; however, a slight decrease in intensity is seen with increasing inter-pulse at 1 Torr and 10 Torr air. In Fig. 3c, the excitation temperature is plotted versus inter-pulse delay for different pressures with air as the bath gas. It is seen here that the inter-pulse delay and the air pressure has little effect on the excitation temperature, with all conditions varying between 7500 and $6300 \mathrm{~K}$.

DP-LIBS in Ar has a similar effect as DP-LIBS in air. In Fig. 4a, DP-LIBS at 630 Torr Ar is compared with SP-LIBS in 630 Torr. DP-LIBS in 630 Torr Ar with an inter-pulse delay of $80 \mu \mathrm{s}$ is $\sim 2.5$-fold greater than SP-LIBS in 630 Torr Ar. Fig. $4 \mathrm{~b}$ plots the peak area from the $481 \mathrm{~nm} \mathrm{Zn} \mathrm{line} \mathrm{versus} \mathrm{inter-pulse} \mathrm{delay} \mathrm{at} \mathrm{varying} \mathrm{Ar} \mathrm{pressures.}$ DP-LIBS has an effect on increasing the spectral intensity in 630 Torr, 300 Torr, and 100 Torr Ar when compared to SP-LIBS. At low Ar pressures of 1 Torr and 10 Torr, the intensity changes very little as the inter-pulse delay is increased. DP-LIBS in 300 Torr and 630 Torr Ar are nearly as effective and seem to provide the most intense spectra when compared to LIBS for all other gases studied. The excitation temperature plotted versus inter-pulse delay at varying Ar pressure (Fig. 4c) shows little change with increase inter-pulse delay and only a slight change depending on the pressure, $\sim 9500 \mathrm{~K}$ for 100 Torr Ar and $\sim 11000 \mathrm{~K}$ for 1 Torr Ar.

The results indicate that DP-LIBS on brass in an atmosphere of 630 Torr Ar gives the most intense spectral intensity for the $\mathrm{Zn}$ line at $481 \mathrm{~nm}$ when compared to spectra 
acquired in air. At low pressures of 1 Torr and 10 Torr DP-LIBS resulted in no increase in spectral intensity for either Ar or air compared to SP-LIBS. These results are consistent with those reported by Hafez et al. [46] looked at SP-LIBS in lower pressures of Ar (1-7 Torr) and observed that signal increases with increasing Ar pressures. While higher pressures result in more collisions with the buffer gas, which decreases plasma lifetime, the observed enhancement was attributed to energy transfer from the buffer gas to analyte. Another reason that Ar provides stronger signal intensities than air may be increased plasma confinement [47] and it is more inert than air [54]. The plasma temperatures for air and Ar are within the range of reported values $[26,55,37,25]$. The lack of variation with inter-pulse delay is likely due to the long delay times used in the current study.

While the Ar and air data are rather well-behaved and consistent with literature reports, the results in with a helium buffer gas are somewhat erratic. Results of DP-LIBS in He are different than those observed for DP-LIBS in Ar or air in that DP-LIBS in He did not result in the most intense spectra. Therefore, Fig. 5 compares SP-LIBS in 100 Torr He (which is the most intense in He) with SP-LIBS in 630 Torr He. SP-LIBS in 100 Torr results in a $\sim 7$ fold increase in $481 \mathrm{~nm} \mathrm{Zn}$ intensity when compared to SP-LIBS at 630 Torr He. As can be seen in Fig. 5b, DP-LIBS seems to have no positive effect on spectral intensity as the inter-pulse delay is increased, except for DP-LIBS at 300 Torr and 630 Torr that showed only a modest increase. DP-LIBS with a $30 \mu$ s inter-pulse delay in 100 Torr He results in a significant decrease in intensity and could be the result of the first pulse perturbing the second (analytical) pulse. In a similar result as the other gases, DP-LIBS at low pressure of He (1 Torr and 10 Torr) has nearly no effect on the 
spectral intensity. Fig. 5c shows the excitation temperature measurements versus interpulse delay in varying He pressure. The excitation temperature in 1 and 10 Torr He showed very little change as the inter-pulse delay was increased and ranged between $9800 \mathrm{~K}$ and $11000 \mathrm{~K}$. At 100 Torr He, the temperate was relatively constant, varying only slightly around $17600 \mathrm{~K}$. At 630 Torr He, the maximum excitation temperature of $\sim 25000 \mathrm{~K}$ was seen with an inter-pulse delay of $10 \mu$ s, while a minimum of temperature of $\sim 18500 \mathrm{~K}$ was observed with $0 \mu$ s inter-pulse delay. At 300 Torr He, the excitation temperature varied wildly and had no clear trend.

One possible explanation for the He observations would be if the plume expanded out of the FOV, which is important as the pressure is decreased [56]. Because plume expansion at reduced pressures likely becomes less spherical and more ellipsoidal [52], the experimental light collecting arrangement may be more critical to homogeneously capturing the plasma emission. For a pressure of 0.1 mbar ( 0.075 Torr), Herrera et al. [57] found a radius of $0.24 \mathrm{~cm}$ and an axial expansion of $0.29 \mathrm{~cm}$ with a delay time of 50 ns. Based on results from Yalcin et al. [52], the plume may expand axially to $\sim 5 \mathrm{~mm}$; however, they did not use delays as long as those used in the current study. Even though the same experimental setup was used for all three buffer gases, less plume confinement is expected for the lighter weight He buffer gas. This is also important because the temperature in the plume is not homogeneous and varies with time and pressure [48]. Thus, if the FOV were inadequate, then at lower pressures and longer delay times, the plume might extend beyond the FOV so that only the back end of the plume would have been observed and the results would not be representative of the whole plasma. Based on simulations and experiments, Wineford and co-workers [58,59] suggest that plasma 
asymmetry can have an effect on the observed emission spectra depending on the light collecting geometry or angle of view. Plume asymmetry (i.e., non-spherical) is expected at reduced pressures [52], but can also occur with DP-LIBS [60]. In the current experimental setup, a lens coupled to a fiber optic was used to collect more of the plume, which can be better than only using a fiber optic [59], to give a FOV of $\sim 25 \mathrm{~mm}$ along the axis of the plasma (i.e., normal to the sample surface). It is conceivable that increased expansion in the radial direction was not adequately captured in the helium studies. Future experimental designs should consider spatially resolved data collection [60] or optical arrangements to collect the light emission more efficiently and homogeneously from all dimensions [61].

Analyte signal intensities in the presence of helium have been reported to be erratic. Henry et al. [62] added helium to air and monitored carbon signal intensities. They observed that the carbon signal initially decreased as helium was added until it reached a minimum with a $75 \%$ He concentration. Further addition of He resulted in an increase in signal intensity until a maximum was observed at $100 \%$. However, plots of the peak-to-base $(\mathrm{P} / \mathrm{B})$ and $\mathrm{S} / \mathrm{N}$ versus He concentration revealed monotomic increase for both $\mathrm{P} / \mathrm{B}$ and $\mathrm{S} / \mathrm{N}$. Henry et al. suggest that the presence of He perturbs the plasma conditions, especially decreasing the absorption of laser energy and the electron density. At high concentrations of He, such as those used in the present study, Henry et al. also suggest that metastable helium states could transfer energy to the analyte directly. Therefore, studies using helium as the buffer gas should be explored with additional monitoring for the behavior of He lines. 
The variation is ionization potentials between the buffer gases may also contribute to the observations, especially those in helium. Chen et al. [48] noted that plasma expansion is faster with ionization than without. Argon has an ionization potential of 15.76 eV), while He has the highest ionization potential of all the buffer gases at $24.58 \mathrm{eV}$ [47]. For air, ionization potentials of the primary constituents nitrogen $\left(\mathrm{N}_{2}\right)$ and oxygen $\left(\mathrm{O}_{2}\right)$ are $15.58 \mathrm{eV}$ and $12.06 \mathrm{eV}$ [63], respectively. Besides the ionization potential, the molecular dissociation energy should also be considered when comparing SP-LIBS and DP-LIBS in air [40]. Oxygen further complicates issues because of the potential chemical reactivity with the analyte [64]. While the first LIP loses some of its energy to ionizing the buffer gas, the second LIP expands in the rarefied gas of the first LIP, allowing it to conserve its energy and enabling an increase in emission intensity. When the ionization energy of the buffer gas is really high, the plasma cannot ionize the gas and SP- and DP-LIBS spectra have similar intensity, as in the case of helium.

\section{Conclusion}

By manipulating the gas composition and pressure along with the use of DPLIBS, the spectral intensity can be increased. In this study, DP-LIBS in an Ar buffer gas provided the most intense spectrum for the $481 \mathrm{~nm}$ line of $\mathrm{Zn}$, which is a minor component in brass, than background gases of air or helium. The results also suggest that the use of a DP-LIBS method in a He environment would provide no benefit; however, it should be noted that the calculated plasma temperatures in He were less consistent than those in Ar or air. In addition, DP-LIBS at reduced pressures less than $\sim 100$ Torr 
generally had no positive effect with the inter-pulse delays explored in this study. The experimental configuration of these studies involved collecting plasma radiation orthogonally to the expansion of the plume, similar to previous DP-LIBS experiments at atmospheric pressures. However, this configuration may not be the most effective for uniformly collecting light from the plume. Future work should include comparison of the results in this study with a similar study that collects plasma radiation more efficiently and homogeneously.

\section{Acknowledgement}

Work supported by the U.S. Department of Energy through the INL Laboratory Directed Research \& Development (LDRD) Program under DOE Idaho Operations Office Contract DE-AC07-05ID1417.

\section{References}

1. De Giacomo A, Dell'Aglio M, De Pascale O, Capitelli M (2007) From single pulse to double pulse ns-Laser Induced Breakdown Spectroscopy under water: Elemental analysis of aqueous solutions and submerged solid samples. Spectroc Acta Pt B-Atom Spectr 62 (8):721-738.

2. Kurniawan KH, Kagawa K (2006) Hydrogen and deuterium analysis using laserinduced plasma spectroscopy. Applied Spectroscopy Reviews 41 (2):99-130. 
3. Lee WB, Wu JY, Lee YI, Sneddon J (2004) Recent applications of laser-induced breakdown spectrometry: A review of material approaches. Applied Spectroscopy Reviews 39 (1):27-97.

4. Martin MZ, Cheng MD, Martin RC (1999) Aerosol measurement by laser-induced plasma technique: A review. Aerosol Science and Technology 31 (6):409-421.

5. Pasquini C, Cortez J, Silva LMC, Gonzaga FB (2007) Laser induced breakdown spectroscopy. Journal of the Brazilian Chemical Society 18 (3):463-512.

6. Sneddon J, Lee YI (1999) Novel and recent applications of elemental determination by laser-induced breakdown spectrometry. Analytical Letters 32 (11):2143-2162.

7. Song K, Lee YI, Sneddon J (1997) Applications of laser-induced breakdown spectrometry. Applied Spectroscopy Reviews 32 (3):183-235.

8. Song K, Lee YI, Sneddon J (2002) Recent developments in instrumentation for laser induced breakdown spectroscopy. Applied Spectroscopy Reviews 37 (1):89-117.

9. Vadillo JM, Laserna JJ (2004) Laser-induced plasma spectrometry: truly a surface analytical tool. Spectroc Acta Pt B-Atom Spectr 59 (2):147-161.

10. Gondal MA, Hussain T, Yamani ZH, Baig MA (2009) On-line monitoring of remediation process of chromium polluted soil using LIBS. Journal of Hazardous Materials 163 (2-3):1265-1271.

11. Hussain T, Gondal MA (2008) Monitoring and assessment of toxic metals in Gulf War oil spill contaminated soil using laser-induced breakdown spectroscopy. Environmental Monitoring and Assessment 136 (1-3):391-399.

12. Kim T, Specht ZG, Vary PS, Lin CT (2004) Spectral fingerprints of bacterial strains by laser-induced breakdown spectroscopy. Journal of Physical Chemistry B 108 (17):5477-5482. 
13. Harmon RS, Remus J, McMillan NJ, McManus C, Collins L, Gottfried JL, DeLucia FC, Miziolek AW (2009) LIBS analysis of geomaterials: Geochemical fingerprinting for the rapid analysis and discrimination of minerals. Applied Geochemistry 24 (6):11251141.

14. Rodriguez-Celis EM, Gornushkin IB, Heitmann UM, Almirall JR, Smith BW, Winefordner JD, Omenetto N (2008) Laser induced breakdown spectroscopy as a tool for discrimination of glass for forensic applications. Anal Bioanal Chem 391 (5):1961-1968.

15. Suliyanti MM, Sardy S, Kusnowo A, Pardede M, Hedwig R, Kurniawan KH, Lie TJ, Kurniawan DP, Kagawa K (2005) Preliminary analysis of C and H in a "Sangiran" fossil using laser-induced plasma at reduced pressure. J Appl Phys 98 (9).

16. Baudelet M, Guyon L, Yu J, Wolf JP, Amodeo T, Frejafon E, Laloi P (2006) Femtosecond time-resolved laser-induced breakdown spectroscopy for detection and identification of bacteria: A comparison to the nanosecond regime. J Appl Phys 99 (8).

17. Osticioli I, Wolf M, Anglos D (2008) An Optimization of Parameters for Application of a Laser-Induced Breakdown Spectroscopy Microprobe for the Analysis of Works of Art. Appl Spectrosc 62 (11):1242-1249.

18. Gaudiuso R, Dell'Aglio M, De Pascale O, Senesi GS, De Giacomo A (2010) Laser Induced Breakdown Spectroscopy for Elemental Analysis in Environmental, Cultural Heritage and Space Applications: A Review of Methods and Results. Sensors 10 (8):7434-7468.

19. Wiens RC, Maurice S (2008) ChemCam's Cost a Drop in the Mars Bucket. Science 322 (5907): 1464-1464.

20. Aragon C, Aguilera JA, Campos J (1993) Determination of carbon content in molten steel using laser-induced breakdown spectroscopy. Appl Spectrosc 47 (5):606-608.

21. Belliveau J, Cadwell L, Coleman K, Huwel L, Griffin H (1985) Laser-induced breakdown spectroscopy of steels at atmospheric-pressure and in air. Appl Spectrosc 39 (4):727-728. 
22. Grant KJ, Paul GL, Oneill JA (1991) Quantitative elemental analysis of iron-ore by laser-induced breakdown spectroscopy. Appl Spectrosc 45 (4):701-705.

23. Millard JA, Dalling RH, Radziemski LJ (1986) Time-resolved laser-induced breakdown spectrometry for the rapid-determination of beryllium in beryllium-copper alloys. Appl Spectrosc 40 (4):491-494.

24. Radziemski LJ, Loree TR, Cremers DA, Hoffman NM (1983) Time-resolved laserinduced breakdown spectrometry of aerosols. Anal Chem 55 (8):1246-1252.

25. Babushok VI, DeLucia FC, Gottfried JL, Munson CA, Miziolek AW (2006) Double pulse laser ablation and plasma: Laser induced breakdown spectroscopy signal enhancement. Spectroc Acta Pt B-Atom Spectr 61 (9):999-1014.

26. Amal K, Elnaby SH, Palleschi V, Salvetti A, Harith MA (2006) Comparison between single- and double-pulse LIBS at different air pressures on silicon target. Appl Phys BLasers Opt 83 (4):651-657.

27. Asgill ME, Brown MS, Frische K, Roquemore WM, Hahn DW (2010) Double-pulse and single-pulse laser-induced breakdown spectroscopy for distinguishing between gaseous and particulate phase analytes. Appl Optics 49 (13):C110-C119.

28. Benedetti PA, Cristoforetti G, Legnaioli S, Palleschi V, Pardini L, Salvetti A, Tognoni E (2005) Effect of laser pulse energies in laser induced breakdown spectroscopy in double-pulse configuration. Spectroc Acta Pt B-Atom Spectr 60 (11):1392-1401.

29. Caneve L, Colao F, Fantoni R, Spizzichino V (2006) Laser ablation of copper based alloys by single and double pulse laser induced breakdown spectroscopy. Applied Physics a-Materials Science \& Processing 85 (2):151-157.

30. Choi SC, Oh MK, Lee Y, Nam S, Ko DK, Lee J (2009) Dynamic effects of a preablation spark in the orthogonal dual-pulse laser induced breakdown spectroscopy. Spectroc Acta Pt B-Atom Spectr 64 (5):427-435. 
31. Galbacs G, Budavari V, Geretovszky Z (2005) Multi-pulse laser-induced plasma spectroscopy using a single laser source and a compact spectrometer. J Anal At Spectrom 20 (9):974-980.

32. Hohreiter V, Hahn DW (2005) Dual-pulse laser induced breakdown spectroscopy: Time-resolved transmission and spectral measurements. Spectroc Acta Pt B-Atom Spectr 60 (7-8):968-974.

33. Scaffidi J, Pender J, Pearman W, Goode SR, Colston BW, Carter JC, Angel SM (2003) Dual-pulse laser-induced breakdown spectroscopy with combinations of femtosecond and nanosecond laser pulses. Appl Optics 42 (30):6099-6106.

34. Stratis DN, Eland KL, Angel SM (2000) Enhancement of aluminum, titanium, and iron in glass using pre-ablation spark dual-pulse LIBS. Appl Spectrosc 54 (12):17191726.

35. Windom BC, Diwakar PK, Hahn DW (2006) Dual-pulse laser induced breakdown spectroscopy for analysis of gaseous and aerosol systems: Plasma-analyte interactions. Spectroc Acta Pt B-Atom Spectr 61 (7):788-796.

36. Noll R, Sattmann R, Sturm V, Winkelmarm S (2004) Space- and time-resolved dynamics of plasmas generated by laser double pulses interacting with metallic samples. J Anal At Spectrom 19 (4):419-428.

37. Sattmann R, Sturm V, Noll R (1995) Laser-induced breakdown spectroscopy of steel samples using multiple Q-switch Nd-YAG Laser-pulses. Journal of Physics D-Applied Physics 28 (10):2181-2187.

38. Bogaerts A, Chen Z, Autrique D (2008) Double pulse laser ablation and laser induced breakdown spectroscopy: A modeling investigation. Spectroc Acta Pt B-Atom Spectr 63 (7):746-754.

39. Scaffidi J, Angel SM, Cremers DA (2006) Emission enhancement mechanisms in dual-pulse LIBS. Anal Chem 78 (1):24-32. 
40. De Giacomo A, Dell'Aglio M, Bruno D, Gaudiuso R, De Pascale O (2008)

Experimental and theoretical comparison of single-pulse and double-pulse laser induced breakdown spectroscopy on metallic samples. Spectroc Acta Pt B-Atom Spectr 63 (7):805-816.

41. Kazakov AY, Gornushkin IB, Omenetto N, Smith BW, Winefordner JD (2006) Radiative model of post-breakdown laser-induced plasma expanding into ambient gas. Appl Optics 45 (12):2810-2820.

42. Bailini A, Ossi PM, Rivolta A (2007) Plume propagation through a buffer gas and cluster size prediction. Appl Surf Sci 253 (19):7682-7685.

43. Galbacs G, Jedlinszki N, Herrera K, Omenetto N, Smith BW, Winefordner JD (2010) A Study of Ablation, Spatial, and Temporal Characteristics of Laser-Induced Plasmas Generated by Multiple Collinear Pulses. Appl Spectrosc 64 (2):161-172.

44. Effenberger Jr AJ, Scott JR (2010) Effect of atmospheric conditions on LIBS spectra. Sensors 10 (5):4907-4925.

45. Iida Y (1990) Effects of atmosphere on laser vaporization an excitation processes of solid samples. Spectroc Acta Pt B-Atom Spectr 45 (12):1353-1367.

46. Hafez MA, Khedr MA, Elaksher FF, Gamal YE (2003) Characteristics of Cu plasma produced by a laser interaction with a solid target. Plasma Sources Sci Technol 12 (2):185-198.

47. Bogaerts A, Chen ZY, Bleiner D (2006) Laser ablation of copper in different background gases: comparative study by numerical modeling and experiments. J Anal At Spectrom 21 (4):384-395.

48. Chen ZY, Bleiner D, Bogaerts A (2006) Effect of ambient pressure on laser ablation and plume expansion dynamics: A numerical simulation. J Appl Phys 99 (6). 
49. Sdorra W, Niemax K (1992) Basic Investigations for Laser Microanalysis .3. Application of Different Buffer Gases for Laser-Produced Sample Plumes. Mikrochim Acta 107 (3-6):319-327.

50. Bailini A, Ossi PM (2007) Expansion of an ablation plume in a buffer gas and cluster growth. Epl 79 (3).

51. Cowpe JS, Astin JS, Pilkington RD, Hill AE (2007) Application of Response Surface Methodology to laser-induced breakdown spectroscopy: Influences of hardware configuration. Spectrochimica Acta Part B: Atomic Spectroscopy 62 (12):1335-1342.

52. Yalcin S, Tsui YY, Fedosejevs R (2004) Pressure dependence of emission intensity in femtosecond laser-induced breakdown spectroscopy. J Anal At Spectrom 19 (10):12951301.

53. Hanafi M, Omar MM, Gamal Y (2000) Study of laser-induced breakdown spectroscopy of gases. Radiation Physics and Chemistry 57 (1):11-20.

54. Babushok VI, DeLucia FC, Dagdigian PJ, Miziolek AW (2005) Experimental and kinetic modeling study of the laser-induced breakdown spectroscopy plume from metallic lead in argon. Spectroc Acta Pt B-Atom Spectr 60 (7-8):926-934.

55. Mao XL, Wen SB, Russo RE (2007) Time resolved laser-induced plasma dynamics. Applied Surface Science 253 (15):6316-6321.

56. Brennetot R, Lacour JL, Vors E, Rivoallan A, Vailhen D, Maurice S (2003) Mars analysis by laser-induced breakdown spectroscopy (MALIS): Influence of mars atmosphere on plasma emission and study of factors influencing plasma emission with the use of Doehlert designs. Appl Spectrosc 57 (7):744-752.

57. Herrera KK, Tognoni E, Gornushkin IB, Omenetto N, Smith BW, Winefordner JD (2009) Comparative study of two standard-free approaches in laser-induced breakdown spectroscopy as applied to the quantitative analysis of aluminum alloy standards under vacuum conditions. J Anal At Spectrom 24 (4):426-438. 
58. Gorbatenko AA, Labutin TA, Popov AM, Zorov NB (2006) Reduction of the matrix influence on analytical signal in laser-enhanced ionization spectrometry with laser sampling. Talanta 69 (4):1046-1048.

59. Shabanov SV, Gornushkin IB, Winefordner JB (2008) Radiation from asymmetric laser-induced plasmas collected by a lens or optical fiber. Appl Optics 47 (11):17451756.

60. Mao XL, Zeng XZ, Wen SB, Russo RE (2005) Time-resolved plasma properties for double pulsed laser-induced breakdown spectroscopy of silicon. Spectroc Acta Pt BAtom Spectr 60 (7-8):960-967.

61. Scott JR, Ham JE, Durham B, Tremblay PL (2004) Ruthenium trisbipyridine as a candidate for gas-phase spectroscopic studies in a Fourier transform mass spectrometer. Spectr-Int J 18 (2):387-396.

62. Henry CA, Diwakar PK, Hahn DW (2007) Investigation of helium addition for laserinduced plasma spectroscopy of pure gas phase systems: Analyte interactions and signal enhancement. Spectroc Acta Pt B-Atom Spectr 62 (12):1390-1398.

63. Samson JAR, Gardner JL (1975) Ionization-Potential of Molecular-Oxygen. Can J Phys 53 (19):1948-1952.

64. Gleason RL, Hahn DW (2001) The effects of oxygen on the detection of mercury using laser-induced breakdown spectroscopy. Spectroc Acta Pt B-Atom Spectr 56 (4):419-430. 


\section{Figures \& Captions}

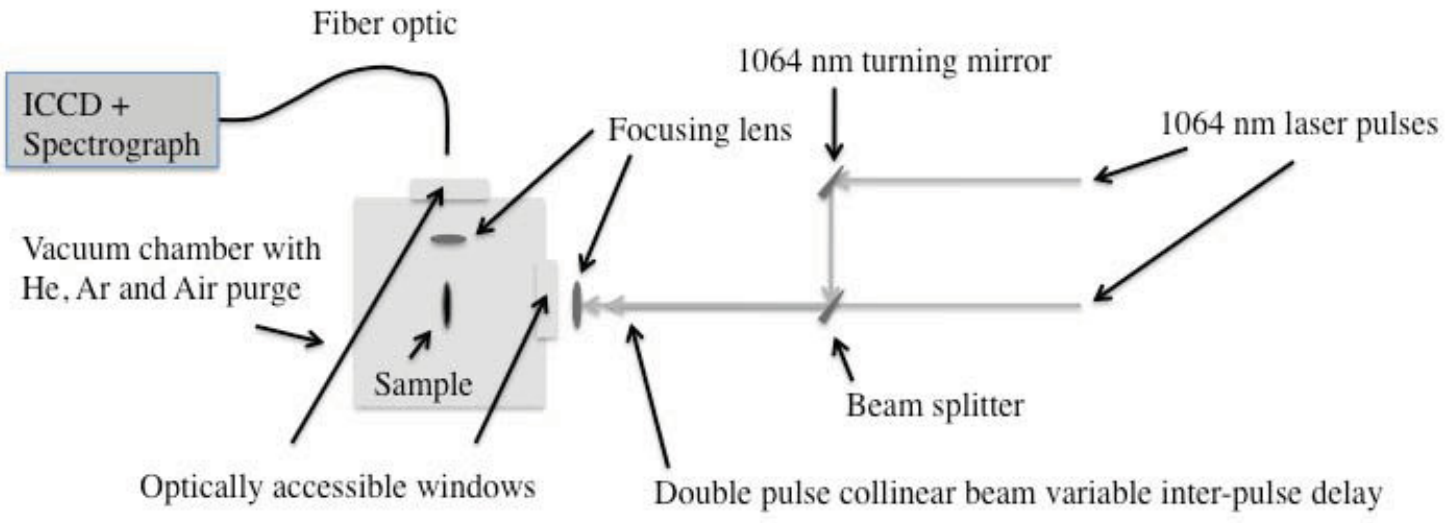

Fig. 1 Experimental set-up for collinear DP-LIBS with variable atmospheres. 


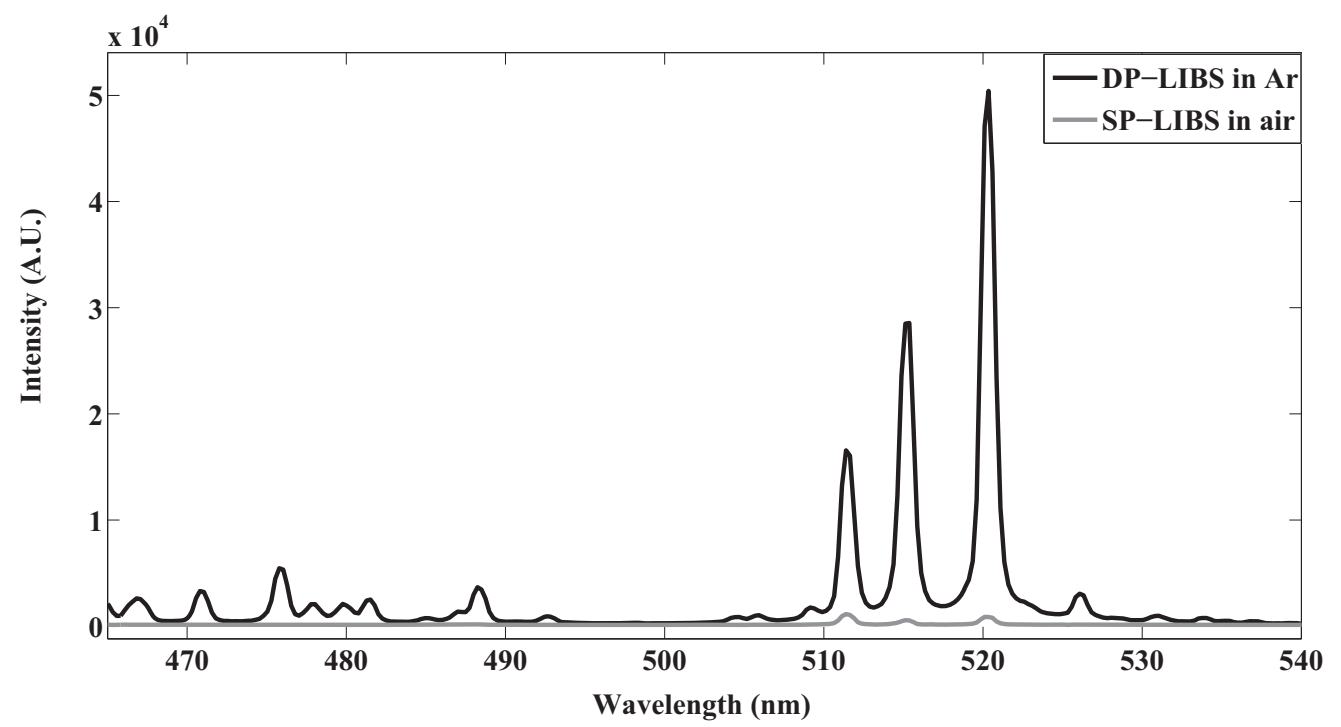

Fig. 2 DP-LIBS with a $80 \mu$ s inter-pulse delay in 630 Torr Ar compared with SP-LIBS in 630 Torr air. 

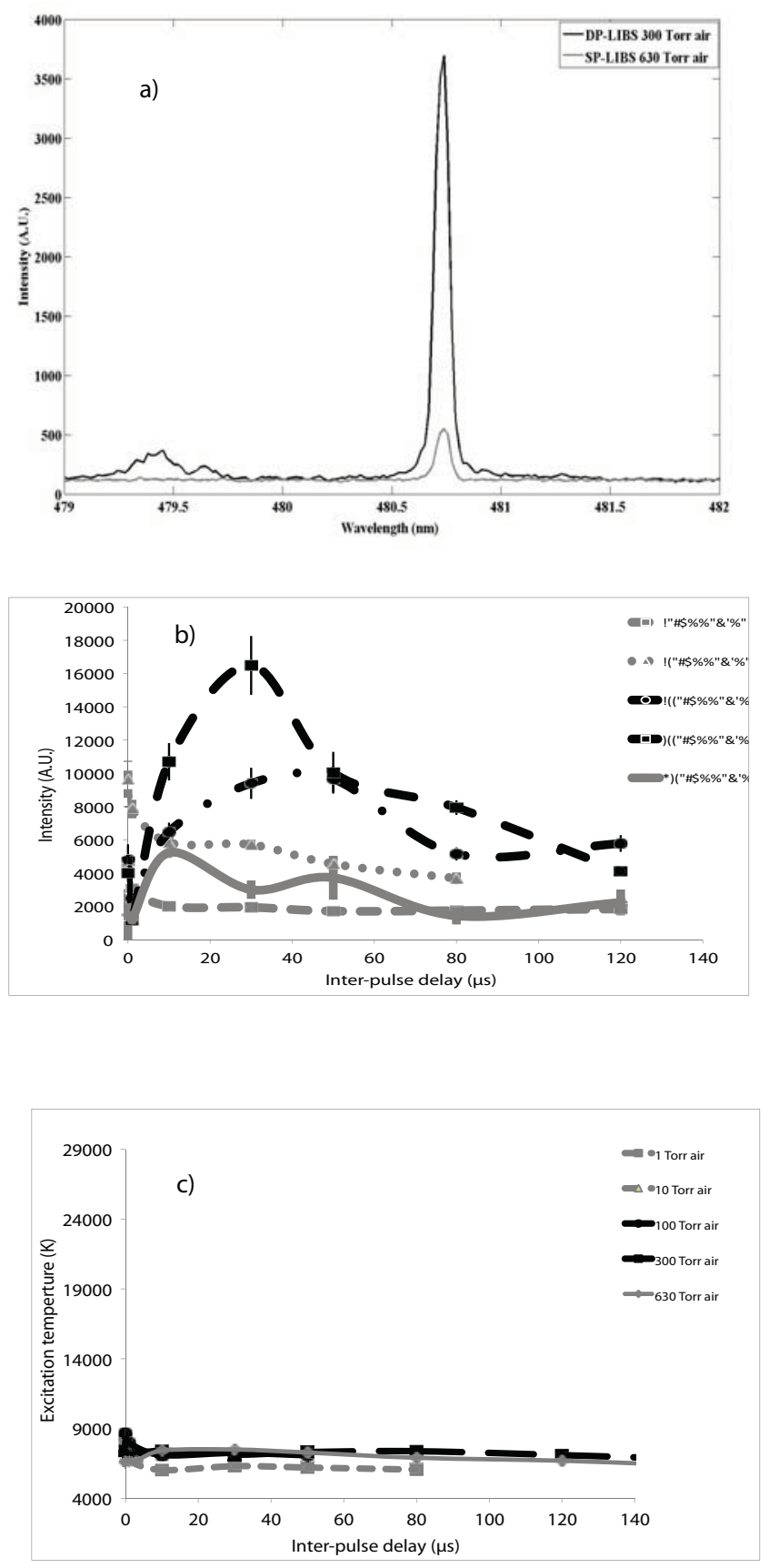

Fig. 3 a) DP-LIBS in 300 Torr air with a $30 \mu$ s inter-pulse delay compared to SPLIBS in 630 Torr air, b) Effect of DP-LIBS on spectral intensity with increasing interpulse delay and at varying air pressures, c) Effect of DP-LIBS on excitation temperature with increasing inter-pulse delay and at varying air pressures (SP-LIBS occurs with $0 \mu \mathrm{s}$ inter-pulse delay). 

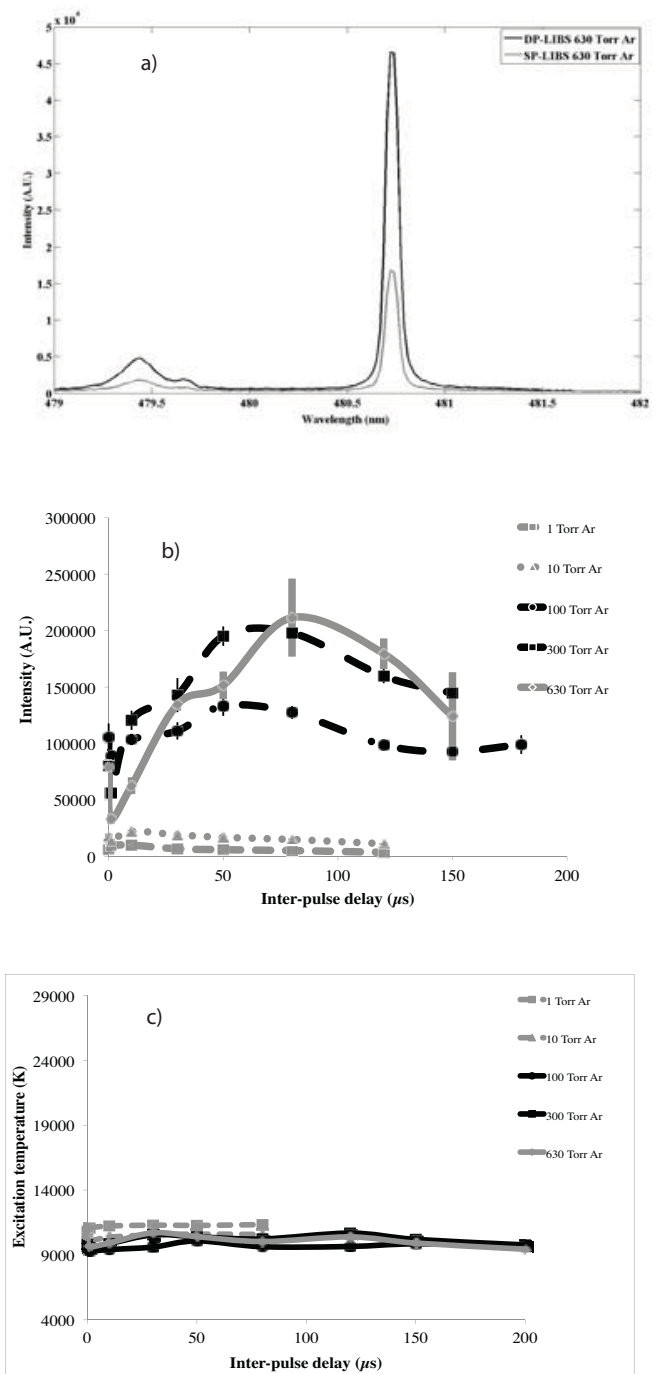

Fig. 4 a) DP-LIBS at 630 Torr Ar with a $80 \mu$ s inter-pulse delay compared to SPLIBS also at 630 Torr Ar, b) Effect of DP-LIBS on spectral intensity with increasing inter-pulse delay at varying Ar pressures, c) Effect of DP-LIBS on excitation temperature with increasing inter-pulse delay and at varying Ar pressures (SP-LIBS occurs with $0 \mu \mathrm{s}$ inter-pulse delay). 

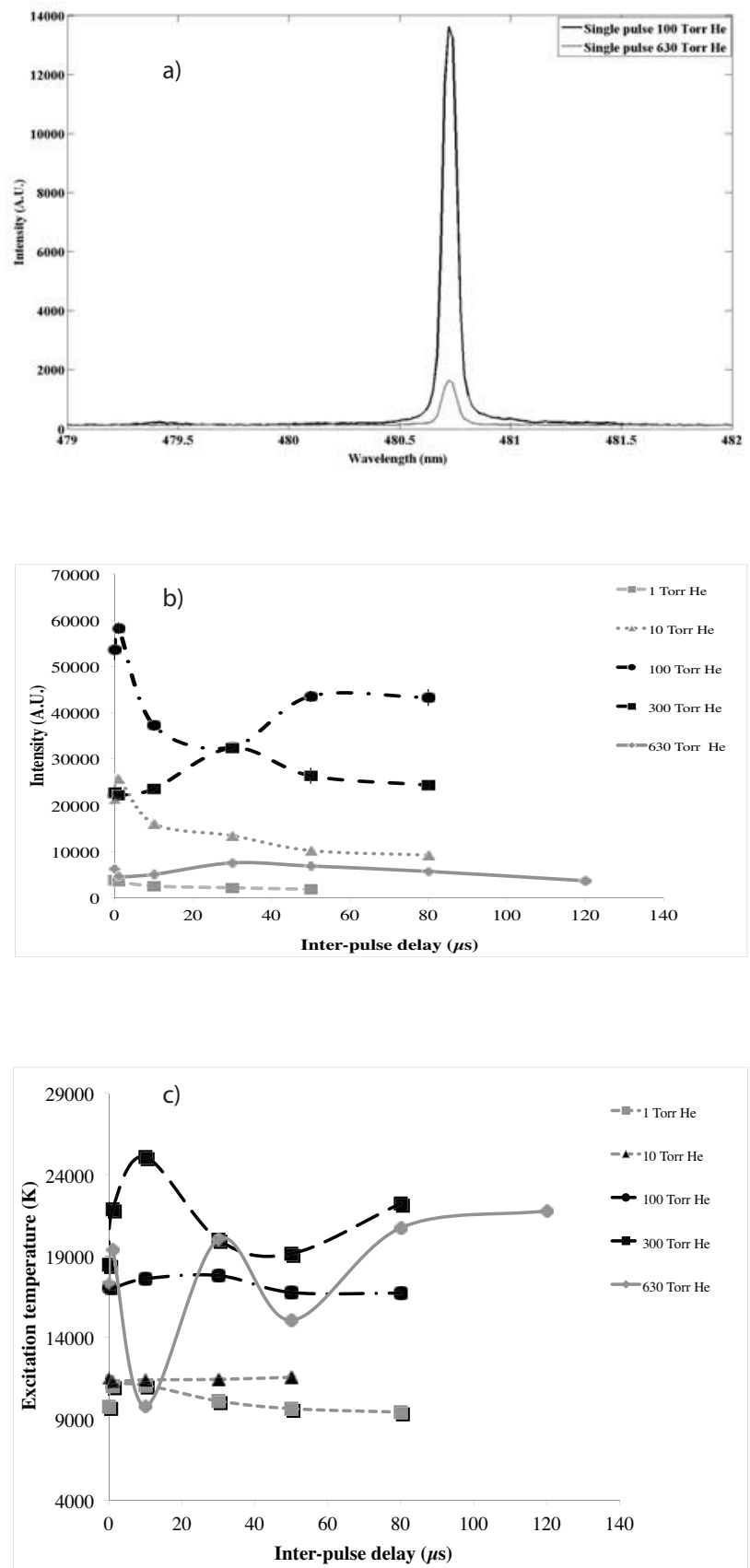

Fig. 5 a) SP-LIBS at 100 Torr compared to SP-LIBS in 630 Torr in He, b) Effect of DP-LIBS with increasing inter-pulse delay with varying He pressure, c) Effect of DPLIBS on excitation temperature with increasing inter-pulse delay and at varying $\mathrm{He}$ pressures (SP-LIBS occurs with $0 \mu$ s inter-pulse delay). 


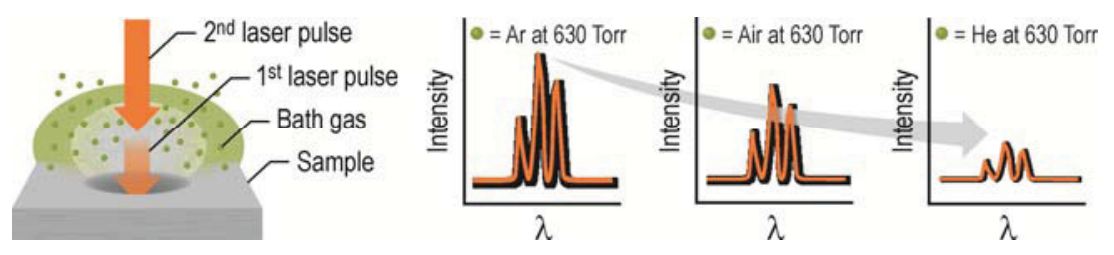

Online Abstract Figure 